\title{
Enhancement of cell proliferation in various mammalian cell lines by gene insertion of a cyclin-dependent kinase homolog Pratik Jaluria ${ }^{1,2}$, Michael Betenbaugh ${ }^{1}$, Konstantinos Konstantopoulos ${ }^{1}$ and Joseph Shiloach*2
}

Address: ${ }^{1}$ Department of Chemical and Biomolecular Engineering, Johns Hopkins University. 221 Maryland Hall, 3400 North Charles Street, Baltimore, MD 21218 USA and 2National Institute of Diabetes \& Digestive \& Kidney Diseases, National Institutes of Health, Biotechnology Unit, Building 14A, Room 170, Bethesda, MD 20892, USA

Email: Pratik Jaluria - pratikj@mail.nih.gov; Michael Betenbaugh - beten@jhu.edu; Konstantinos Konstantopoulos - kkonsta1@jhu.edu; Joseph Shiloach* - yossi@nih.gov

* Corresponding author

Published: 18 October 2007

BMC Biotechnology 2007, 7:7| doi:|0.|I86/|472-6750-7-7|
Received: 5 June 2007

Accepted: 18 October 2007

This article is available from: http://www.biomedcentral.com/I472-6750/7/7I

(c) 2007 Jaluria et al; licensee BioMed Central Ltd.

This is an Open Access article distributed under the terms of the Creative Commons Attribution License (http://creativecommons.org/licenses/by/2.0), which permits unrestricted use, distribution, and reproduction in any medium, provided the original work is properly cited.

\begin{abstract}
Background: Genomics tools, particularly DNA microarrays, have found application in a number of areas including gene discovery and disease characterization. Despite the vast utility of these tools, little work has been done to explore the basis of distinct cellular properties, especially those important to biotechnology such as growth. And so, with the intent of engineering cell lines by manipulating the expression of these genes, anchorage-independent and anchorage-dependent HeLa cells, displaying markedly different growth characteristics, were analyzed using DNA microarrays.

Results: Two genes, cyclin-dependent kinase like 3 (cdk/3) and cytochrome c oxidase subunit (cox/5), were up-regulated in the faster growing, anchorage-independent (suspension) HeLa cells relative to the slower growing, anchorage-dependent (attached) HeLa cells. Enhanced expression of either gene in the attached HeLa cells resulted in elevated cell proliferation, though insertion of $c d k / 3$ had a greater impact than that of $\operatorname{cox} 15$. Moreover, flow cytometric analysis indicated that cells with an insert of $c d k / 3$ were able to transition from the G0/GI phases to the $S$ phase faster than control cells. In turn, expression of cox 15 was seen to increase the maximum viable cell numbers achieved relative to the control, and to a greater extent than cdk/3. Quantitatively similar results were obtained with two Human Embryonic Kidney-293 (HEK-293) cell lines and a Chinese Hamster Ovary (CHO) cell line. Additionally, HEK-293 cells secreting adipocyte complement-related protein of $30 \mathrm{kDa}$ (acrp30) exhibited a slight increase in specific protein production and higher total protein production in response to the insertion of either $c d k / 3$ or cox 15 .

Conclusion: These results are consistent with previous studies on the functionalities of $c d k / 3$ and $\operatorname{cox} / 5$. For instance, the effect of $c d k / 3$ on cell growth is consistent with its homology to the $c d k 3$ gene which is involved in $\mathrm{GI}$ to $\mathrm{S}$ phase transition. Likewise, the increase in cell viability due to cox 15 expression is consistent with its role in oxidative phosphorylation as an assembly factor for cytochrome coxidase and its involvement removing apoptosis-inducing oxygen radicals. Collectively, the present study illustrates the potential of using microarray technology to identify genes influential to specific cellular processes with the possibility of engineering cell lines as desired to meet production needs.
\end{abstract}




\section{Background}

The properties of a cell line being used to produce therapeutic or diagnostic compounds are vital elements in designing a production process [1-3]. Such properties include: nutritional requirements, adhesion capabilities, glycosylation capabilities, and cellular response to stimuli $[1,2]$. Perhaps one of the most important cellular properties is proliferation, referring to the rate at which cells grow and divide by passing through the different cell cycle phases $[3,4]$.

The ability to alter specific properties of a cell line would undoubtedly find application in the field of biotechnology. Currently, protocols and technical notes are readily available to adapt specific cell lines to make them more suitable for a particular process. These procedures have focused primarily on screening cell populations to identify and subsequently select cells with the desired feature [3-5]. Less emphasis has been placed on either identifying how a particular adaptation occurs or the ability to engineer the desired modification directly into the cell lines.

Previous studies exploring proliferation have examined the roles of various genes and/or chemicals regulating the cell cycle in hopes of indirectly stimulating cellular growth $[6,7]$. For example, it was shown that transfection of the gene $c-m y c$, a proto-oncogene that encodes a transcription factor, can enhance the growth rate of Chinese Hamster Ovary (CHO) cells [8]. Other studies have focused on deciphering the sequence of events necessary for cells to complete the cell cycle by targeting specific genes $[1,9]$. It has been found that certain proteins are necessary for cells to maintain viability and/or function such as growth factors and stress sensors [10]. Some studies have identified groups or families of proteins that play important roles maintaining cellular function such as members of the serine/threonine protein kinase family; essential for phase transitions of the cell cycle [10]. Based on this, a number of studies have explored what other proteins associate with members of this kinase family such as cyclins, inhibitors, and ubiquitin $[4,10]$. However, the complexity of the cell cycle has limited the way in which mammalian cells can be manipulated to increase their growth.

In the present study, complementary DNA (cDNA) microarrays were used to identify genes potentially influencing cellular growth by comparing a slow growing, anchoragedependent (attached) cell line to a fast growing, anchorage-independent (suspension) cell line. Both cell lines were HeLa cell lines [11]. Microarray data were probed for differentially expressed genes using clustering algorithms and proposed functionalities. Two genes, $c d k l 3$ [GenBank: NM016508] and cox15 [GenBank: NM078470], had higher expression in the fast growing, suspension HeLa cells than in the slow growing, attached HeLa cells. The cDNA for both genes were transfected into a number of cell lines including Human Embryonic Kidney-293 (HEK293), CHO, and Madin-Darby Canine Kidney (MDCK). The growth characteristics of these cell lines were monitored along with recombinant protein production levels in the HEK-293 ACRP30 (adipocyte complement-related protein of $30 \mathrm{kDa}$ ) cell line using ELISA. Further characterization of the transfected cell physiology was performed using flow cytometry analysis.

\section{Results}

\section{Growth of HeLa cells in bioreactors}

The attached and suspension HeLa cell lines were grown in bioreactors in three independent experiments using the same media and culture environment. The attached HeLa cells had to be grown using microarriers [12]. The measured viable cell densities for both cells lines are shown in Figure 1. The suspension HeLa cells grew at a maximum specific growth rate of $0.038 \pm 0.002 \mathrm{~h}^{-1}, 40 \%$ higher than the attached HeLa cells which grew at a maximum specific growth rate of $0.027 \pm 0.001 \mathrm{~h}^{-1}$. The suspension HeLa cells achieved a maximum viable cell density of about 3.5 $\times 10^{6}$ cells $/ \mathrm{mL}$, twice the maximum viable cell density obtained for the attached HeLa cells. The overall viability for each cell line was above $90 \%$ until the stationary phase of growth was reached.

\section{Comparison of gene transcription levels between suspension and attached HeLa cells}

Samples for microarray analysis were taken at the same growth phases from each bioreactor, at regular intervals. Imaging software was used to determine which comparisons exhibited the most significant differences between the two cell lines and had the highest levels of overall tran-

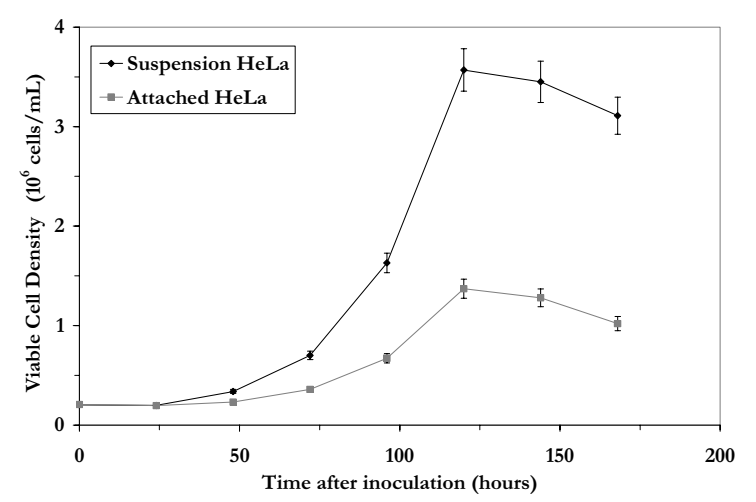

Figure I

Growth of suspension and attached HeLa cells in bioreactors under batch conditions. Attached HeLa cells were grown on Cytodex 3 microcarriers. 
scriptional activity. Samples from the middle exponential growth phase, corresponding to the maximum specific growth rates, were found to meet these criteria. This information was deciphered by examining scanned images of hybridized microarrays using GenePix, a visualization software program.

Analysis of the hybridized cDNA microarrays began with quality control (i.e. removing spots of poor signal) and continued with implementation of total intensity normalization $[13,14]$. The data were filtered by removing genes lacking consistency between slides in terms of how close the expression ratio from any one slide was to the median expression ratio calculated from all of the slides. The data were also screened for genes with expression ratios from the slides in the same direction (i.e. either upregulated or downregulated across all of the slides).

Using clustering algorithms, the genes were organized into groups [14,15]. Principle component analysis (PCA) and gap statistic were used to estimate the number and size of groups inherently present in the data. Results of these algorithms indicated groups of $8,9,13$, or 14 were likely to form. With this information, self-organizing maps (SOMs) and hierarchical clustering were then applied to the data to segregate the genes into distinct groups. These clusters were probed to identify subsets of genes with relevance to cellular growth based on known or proposed functionalities related to cell cycle regulation, apoptosis, and/or signal transduction. Genes that could be categorized in this manner were then interrogated further based on the level of differential expression with a sampling of the results shown in Table 1 . The ratios listed in Table 1 were determined from a sample size of at least 12; based on the number of spots per slide and the number of slides analyzed. Of the genes listed, $c d k l 3$ [GenBank: NM016508] and cox15 [GenBank: NM078470] were selected for further analysis. As shown in Figure 2, both $c d k l 3$ and $c o x 15$ had expression levels greater than 1 , indicating higher expression in the suspension HeLa cell line than in the attached HeLa cell line.

\section{Verification of microarray results}

As can be seen in Table 1 and Figure 2, the expression ratios of both $c d k l 3$ and cox 15 varied over the different samples, establishing the need to verify these results. For this purpose, a series of RT-PCR experiments were conducted, the results of which are shown in Table 2 along with two controls,gapd and pgk1. The findings in the Table demonstrate the expression levels are consistent between the microarray data and the RT-PCR experiments.

\section{Enhanced expression of cdkl3 and cox I5 in attached HeLa cells}

To evaluate the impact of these two genes in cell culture, DNA plasmids containing either $c d k l 3$ or cox 15 were transfected into the attached HeLa cells. Cells transfected with only blank plasmids (i.e. containing neither $c d k l 3$ nor cox15) served as the control. Each plasmid also contained the neomycin gene allowing cells to be selected based on resistance to geneticin. A number of clones were selected in this manner over the course of 1-2 weeks (post-transfection) and then assayed for expression of the translated protein using western blots. Figure 3 illustrates the protein expression of cells that have gone through this selection process. These blots indicate cells transfected with either cdkl3 or cox15 had significantly higher protein levels than the control cells. It should be noted that when the selection marker is not present in the culture media, these cell lines return to normal protein production levels within 56 passages.

The growth profiles/characteristics of the attached HeLa cells transfected with $c d k l 3$, cox 15 , or a blank plasmid are summarized in Table 3 . All three cell lines were grown on microcarriers in $250 \mathrm{~mL}$ spinner flasks. The maximum specific growth rates, determined in three independent experiments, revealed that the attached HeLa cells trans-

Table I: Gene names and maximum/minimum expression ratios for differentially expressed genes identified following normalization and application of clustering algorithms. Expression ratios are of the form: suspension HeLa/attached HeLa

\begin{tabular}{clcc}
\hline Gene Symbol & \multicolumn{1}{c}{ Gene Name } & Maximum Expression Ratio & Minimum Expression Ratio \\
\hline cdkl3 & cyclin-dependent kinase-like 3 & 3.32 & 1.76 \\
coxl5 & cytochrome c oxidase assembly protein & 3.87 & 1.95 \\
agpat2 & I-acylglycerol-3-phosphate O-acyltransferase 2 & 3.13 & 1.64 \\
fgf7 & Fibroblast growth factor 7 & 3.50 & 1.75 \\
dtymk & deoxythymidylate kinase (thymidylate kinase) & 4.05 & 1.92 \\
arhf & ras homolog gene family f & 2.95 & 1.70 \\
plagll & pleiomorphic adenoma gene-like I & 0.04 & 0.02 \\
tiall & cytotoxic granule-associated RNA binding protein-like & 0.68 & 0.14 \\
& I & & \\
\hline
\end{tabular}

Note: Each cDNA slide analyzed had at least 3 spots for each of the genes listed above. Analysis of 4 slides translated to a total of at least 12 ratios for each gene. Both the maximum and the minimum expression ratios per gene were determined from these 12 ratios (i.e. sample size). 


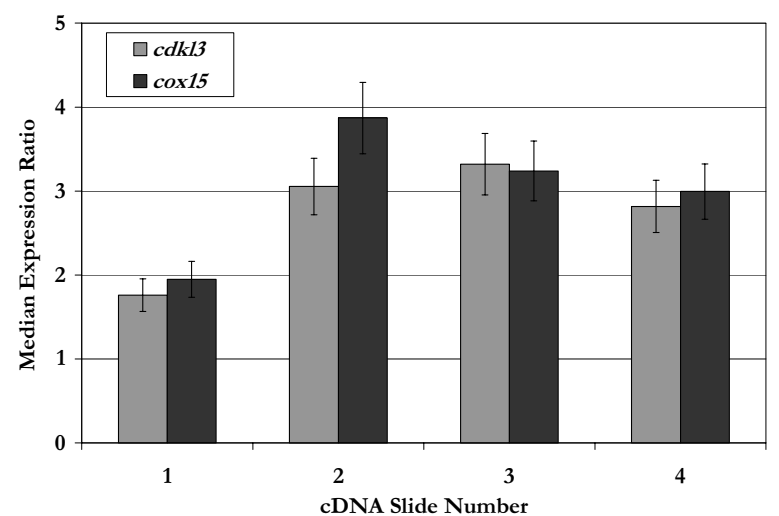

Figure 2

Median expression ratios for $c d k / 3$ and cox 15 from four different spotted cDNA microarray slides. The median expression ratio is calculated from 3 or more gene-specific spots on a single slide. The error bars indicate the range in expression ratios observed for a given slide.

fected with a plasmid containing either $c d k l 3$ or $\operatorname{cox} 15$ grew faster than the control cells. Cells expressing $c d k l 3$ had a maximum specific growth rate $20 \%$ greater than the control cells while the cells expressing cox 15 had a maximum specific growth rate $15 \%$ greater than the control cells. Using a t-test, it was determined that the differences in maximum specific growth rates between treated and control cells were statistically significant for $\alpha$-values, also referred to as risk level (i.e. the chance associated with finding a statistically significant difference between means when there is none) as low as 0.005 . It was also observed that the duration of the lag phase in cells expressing either cdkl3 or cox 15 was $5-8 \%$ shorter than that of the control cells.

Cells expressing cox15 were also able to achieve a maximum viable cell density $11 \%$ higher than the control cells whereas cells expressing $c d k l 3$ achieved a maximum viable cell density equivalent to the control cells (Table 3 ). The difference between cells expressing cox 15 and control cells were statistically significant for $\alpha$-values as low as 0.005 . Similar results, in terms of growth rates and cell densities, were obtained when these cell lines were grown in $\mathrm{T}$ - flasks. No additive effects were observed when both genes were simultaneously expressed (data not shown).

\section{Gene sequence homology and enhanced expression of cdkl3 and cox 15 in other cell lines}

Using two online databases, Harvester and GenBank, the nucleotide sequences for both $c d k l 3$ and cox 15 were determined $[16,17]$. Sequence analysis was performed to quantify homology between varying species. For the $c d k l 3$ gene, the following were determined: $94 \%$ similarity with Canis familiaris (dog), 83\% similarity with Rattus norvegicus (rat), and 90\% similarity with Mus musculus (mouse). Slightly less similar was the cox 15 gene with $87 \%$ similarity to Canis familiaris (dog), $86 \%$ similarity to Rattus norvegicus (rat), and $86 \%$ similarity to Mus musculus (mouse). Based on these results, three additional cell lines, HEK293, CHO, and MDCK were chosen for investigation [18]. The HEK-293 cells were selected to investigate whether or not the genes identified using HeLa cells had functionality in other human-derived cell lines. With significant homology between the two rodent species in terms of gene transcripts, $\mathrm{CHO}$ cells were chosen due to their widespread use in commercial applications [19]. However, without a central database detailing the sequence of the CHO genome, the existence of homologs for either $c d k l 3$ or cox15 could not be verified in that species. The canine cell line selected, MDCK, is commonly used in the production of vaccines [19].

When grown under the same spinner flask conditions previously described, two different types of HEK-293 cells (HEK-293 and HEK-293 ACRP30) expressing either $c d k l 3$ or cox15 grew faster than the control cells (Table 3). The maximum specific growth rate of the HEK-293 cells expressing $c d k l 3$ was $19 \%$ greater than the control cells; a value statistically significant for $\alpha$-values as low 0.005 . Similarly, the maximum specific growth rate of the HEK293 cells expressing cox 15 was $13 \%$ greater than the control cells; a value statistically significant for $\alpha$-values as low 0.01. Cells expressing cox15 grew to a maximum viable cell density $9 \%$ higher than the control cells; statistically significant for $\alpha$-values as low 0.005 .

HEK-293 ACRP30 cells expressing $c d k l 3$ had a maximum specific growth rate $16 \%$ higher than the control cells (Table 3); significant for $\alpha$-values as low 0.005. These cells

Table 2: Median expression levels for specific genes as determined by CDNA microarrays and RT-PCR. Expression ratios are of the form: suspension HeLa/attached HeLa

\begin{tabular}{cccc}
\hline Gene Symbol & Gene Name & cDNA microarrays & RT-PCR \\
\hline cdkl3 & cyclin-dependent kinase-like 3 & 2.74 & $2.6 \pm 0.2$ \\
coxl5 & cytochrome c oxidase assembly protein & 3.01 & $3.4 \pm 0.3$ \\
gapd & Glyceraldehyde-3-phosphate dehydrogenase & 1.03 & $1.2 \pm 0.1$ \\
Pgkl & Phosphoglycerate kinase I & 0.98 & $1.0 \pm 0.1$ \\
\hline
\end{tabular}




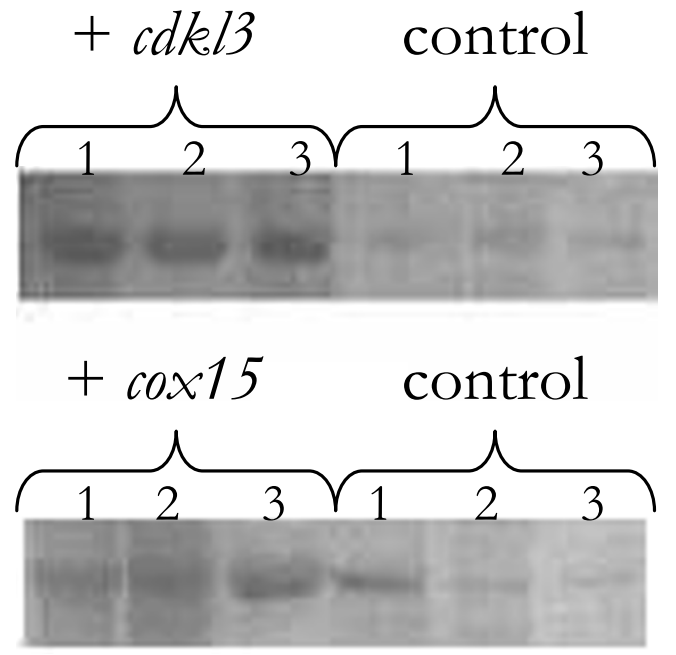

\section{Figure 3}

Western blot analysis for several different cell lines indicating relative expression levels. These cells were derived from 3 different colonies that had already been screened and selected for expression of the transfected plasmid. At the time of analysis, these cells had been grown for over 6 days, post-transfection. The control cells were transfected with blank plasmids. I - HeLa 2 - HEK-293 3 - CHO

expressing cox 15 also had a maximum specific growth rate higher than the control cells, by approximately 14\%; statistically significant for $\alpha$-values as low 0.001 . The HEK-
293 ACRP30 cells achieved a maximum viable cell density $8 \%$ greater than the control cells; statistically significant for $\alpha$-values as low 0.01 .

$\mathrm{CHO}$ cells with inserts of either $c d k l 3$ or cox 15 were also found to grow faster than control cells, as shown in Table 3. CHO cells expressing cdkl3 had a maximum specific growth rate $9 \%$ higher than the control cells; a value statistically significant for $\alpha$-values as low 0.005 . CHO cells expressing cox 15 had a maximum specific growth rate $5 \%$ higher than the control cells; statistically significant for $\alpha$ values as low 0.025. In addition, cells expressing either $c d k l 3$ or $\operatorname{cox} 15$ achieved maximum viable cell densities $5 \%$ ( $\alpha$-value $\geq 0.005$ ) or $7 \%$ ( $\alpha$-value $\geq 0.001$ ) greater than the control cells, respectively. In contrast, MDCK cells with inserts of either $c d k l 3$ or $\operatorname{cox} 15$ did not exhibit differences in terms of growth rates or maximum viable cell densities, as shown in Table 3.

\section{Examining cell proliferation using flow cytometry and ELISA}

In order to evaluate the effects of enhanced expression of either $c d k l 3$ or $\operatorname{cox} 15$ on cell cycle, analysis of cell cycle progression was performed using flow cytometry. The cell line exhibiting the greatest effects of enhanced expression of either $c d k l 3$ or $\operatorname{cox} 15$ was the attached HeLa cell line. In this assay, cells were initially synchronized and then grown up until late into the exponential phase. Cells with enhanced expression of either $c d k l 3$ or cox15, shown in Figure 4, illustrated a lower percentage of cells in the GOG1 phases and a higher percentage in the $S$ phase compared to control cells. The percentage of cells in the G2-M phases was similar between the three cell lines.

Table 3: Growth-related data for varying cell lines grown in spinner flasks

\begin{tabular}{|c|c|c|c|c|c|c|}
\hline \multirow[t]{2}{*}{ Cell Type } & \multirow[t]{2}{*}{ Cell line } & \multicolumn{4}{|c|}{ Maximum growth rate during the exponential growth phase (hours $\left.{ }^{-1}\right)$ from 3 different runs } & \multirow[t]{2}{*}{$\begin{array}{l}\text { Maximum viable cell } \\
\text { density }\left(10^{6} \text { cells } / \mathrm{mL}\right)\end{array}$} \\
\hline & & Run I & Run 2 & Run 3 & Average Change vs. Control (\%) & \\
\hline \multirow[t]{3}{*}{ HeLa } & control & 0.027 & 0.026 & 0.026 & & $1.49 \pm 0.03$ \\
\hline & $+c d k l 3$ & 0.031 & 0.032 & 0.032 & 20 & $1.53 \pm 0.03$ \\
\hline & $+\operatorname{cox} 15$ & 0.030 & 0.030 & 0.031 & 15 & $1.66 \pm 0.02$ \\
\hline \multirow{3}{*}{ HEK-293 } & control & 0.030 & 0.030 & 0.032 & & $1.98 \pm 0.02$ \\
\hline & $+c d k / 3$ & 0.035 & 0.037 & 0.037 & 19 & $2.04 \pm 0.04$ \\
\hline & $+\operatorname{cox} 15$ & 0.034 & 0.036 & 0.034 & 13 & $2.15 \pm 0.05$ \\
\hline \multirow[t]{3}{*}{ HEK-293 ACRP30 } & control & 0.031 & 0.031 & 0.032 & & $1.94 \pm 0.04$ \\
\hline & $+c d k / 3$ & 0.037 & 0.037 & 0.035 & 16 & $2.01 \pm 0.02$ \\
\hline & $+\operatorname{cox} 15$ & 0.036 & 0.035 & 0.036 & 14 & $2.10 \pm 0.06$ \\
\hline \multirow[t]{3}{*}{$\mathrm{CHO}$} & control & 0.033 & 0.034 & 0.034 & & $1.95 \pm 0.01$ \\
\hline & $+c d k / 3$ & 0.036 & 0.037 & 0.037 & 9 & $2.04 \pm 0.02$ \\
\hline & $+\operatorname{cox} 15$ & 0.036 & 0.035 & 0.035 & 5 & $2.09 \pm 0.03$ \\
\hline \multirow[t]{3}{*}{ MDCK } & control & 0.029 & 0.030 & 0.030 & & $1.12 \pm 0.04$ \\
\hline & $+c d k / 3$ & 0.030 & 0.029 & 0.030 & 0 & $1.10 \pm 0.02$ \\
\hline & $+\operatorname{cox} 15$ & 0.028 & 0.029 & 0.028 & -5 & $1.08 \pm 0.07$ \\
\hline
\end{tabular}



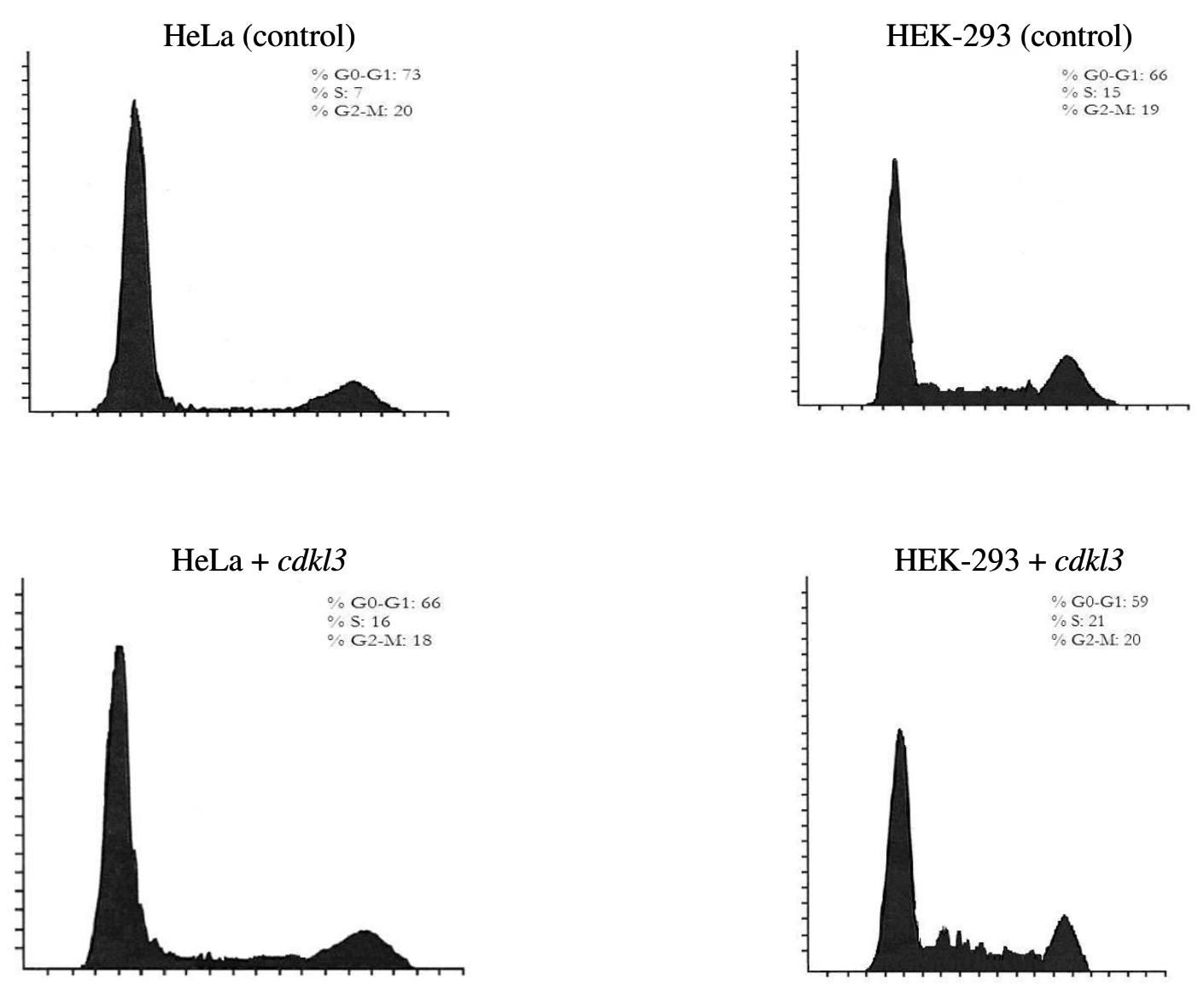

HeLa $+\operatorname{cox} 15$

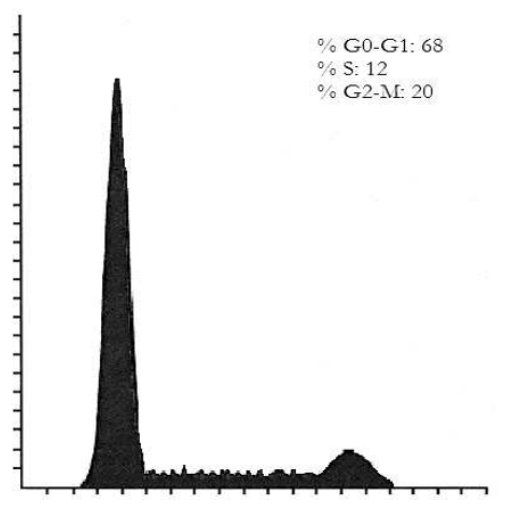

HEK-293+ $\operatorname{cox} 15$

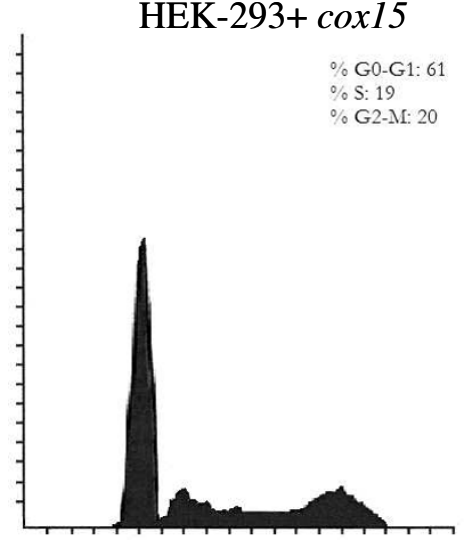

\section{Figure 4}

Results of flow cytometric analysis; cell count vs. fluorescence. In each image the first peak (also the largest peak) indicates cells in the G0/GI phases whereas the second, smaller peak indicates cells in the G2/M phases. In between these two peaks are cells in the $\mathrm{S}$ phase. The percentages of cells in each phase are shown along side each figure. 
To investigate the potential effects of enhanced expression of either $c d k l 3$ or $c o x 15$ on protein production in HEK-293 cells constitutively secreting recombinant adipocyte complement-related protein of $30 \mathrm{kDa}$ (acrp30); HEK-293 ACRP30 were transfected with plasmids containing either cox 15 or $c d k l 3$. The cells were plated in T-flasks at an equivalent seeding density. The total protein production levels in $\mathrm{ng} / \mathrm{mL}$ were measured in each flask along with the productivities of acrp30 on a per cell basis, shown in Table 4 . Cells were assayed during the exponential growth phase and not during the stationary phase, therefore the cell densities listed were not maximum. The total protein levels were increased by $20 \%$ in the cells transfected with cdkl3 while an $11 \%$ increase was seen in cells transfected with cox15. Specific production levels were higher in the cells expressing either $c d k l 3$ or cox 15 relative to control cells.

\section{Discussion}

Using clustering algorithms driven by gene ontology, differentially expressed genes with functionalities relevant to cell growth were identified when comparing slow growing, attached HeLa cells to fast growing, suspension HeLa cells (Table 1). Two of the genes selected for further investigation, $c d k l 3$ and cox15, were expressed at higher levels in the suspension than in the attached HeLa cells, though each gene was thought to relate to cellular growth in a distinct way. Previous studies suggest $c d k l 3$ (cyclin-dependent kinase like 3) is involved in cell cycle regulation/ progression, and cox15 (cytochrome c oxidase assembly protein) is involved in energy metabolism $[20,21]$.

The gene $c d k l 3$ encodes a polypeptide of 455 amino acids titled NKIAMRE [Swiss-Prot: Q8IVW4] that localizes in the cytoplasm and is thought to be a component of a kinase complex that phosphorylates the C-terminus of RNA polymerase II $[20,22,23]$. Although, the precise functionality of NKIAMRE remains unknown, sequence similarities and the presence of several motifs together imply membership to both the mitogen-activated protein kinase (mapk) family and cyclin-dependent kinase (cdk) family $[20,24]$. Both of these families are known to be involved in signaling, cell cycle regulation, migration, and survival [25].

As the gene name suggests, $c d k l 3$ is most similar in sequence to cyclin-dependent kinase $3(c d k 3)$; a gene that encodes a kinase believed to be necessary for the G1-S transition in mammalian cells $[10,26]$. The protein encoded by $c d k l 3$ also contains the highly conserved TXY (Threonine-X-Tyrosine) motif found in the activation loop domains of either a mapk or cdk $[20,24]$. In addition, two residues, a serine and a tyrosine, in subdomain I closely resemble an adenosine triphosphate (ATP) binding domain and a negative regulation site typical of a cdk. The protein's name itself, NKIAMRE signifies an amino acid sequence thought to be a cyclin-binding domain also found in a cdk $[20,22]$. Additionally, the catalytic domain of NKIAMRE contains two highly conserved sequences found in both serine/threonine and tyrosine protein kinases $[20,24]$. High expression of nkiamre was detected in two aggressive types of anaplastic large cell lymphoma (ALCL) tumors when compared to peripheral blood lymphocytes [27]. This finding suggests the gene may also play a role in pathogenesis and/or tumorigenesis.

The gene $c d k l 3$ appears to act as either a cell cycle mimic or regulator with several distinct functions including ATP \& nucleotide binding, kinase activity, and transferase activity; all of which relate to regulation of the cell cycle and control of cellular proliferation. The current study supports these claims and suggests that $c d k l 3$, like its homolog $c d k 3$, influences the cell cycle through observations of increased growth rates, reduced lag phases, and greater protein production. The gene $c d k 3$ is thought to be rate-limiting for mammalian cell cycle progression [28]. Other studies have shown mammalian cells require $c d k 3$ to exit the G0 and G1 phases as well as enter the S phase $[29,30]$. These observations are also supported by the present work through flow cytometry experiments that indicated cells with an insert of $c d k l 3$ were able to transition from the G0/G1 phases to the S phase faster than cells without the insert.

Table 4: ELISA results for HEK-293 ACRP30 cells with and without gene inserts. The results shown are from I of 3 different runs performed, but are representative of all 3 runs

\begin{tabular}{|c|c|c|c|}
\hline Cell line & Viable cell density $\left(10^{6}\right.$ cells $\left./ \mathrm{mL}\right)$ & Total protein production (ng) & $\begin{array}{l}\text { Average specific protein } \\
\text { production }\left(\frac{\mathrm{ng} \cdot \mathrm{mL}}{\text { cells }}\right)\end{array}$ \\
\hline control with plasmid & $1.24 \pm 0.01$ & $1580 \pm 130$ & $1.27 \times 10^{-3}$ \\
\hline$+c d k / 3$ & $1.40 \pm 0.02$ & $1900 \pm 160$ & $1.36 \times 10^{-3}$ \\
\hline$+\operatorname{cox} 15$ & $1.31 \pm 0.03$ & $1750 \pm 190$ & $1.34 \times 10^{-3}$ \\
\hline
\end{tabular}

Note: Each cDNA slide analyzed had at least 3 spots for each of the genes listed above. Analysis of 4 slides translated to a total of at least 12 ratios for each gene. Both the maximum and the minimum expression ratios per gene were determined from these 12 ratios (i.e. sample size). 
The human gene cox15 could relate to cellular growth from the perspective of energy metabolism (i.e. cells growing faster have greater energy demands). It encodes a cytochrome c oxidase (COX) assembly protein referred to as COX15 [Swiss-Prot: Q7KZN9] which localizes in the mitochondrial inner membrane [31,32]. Significant expression of cox 15 has been observed in tissues with high oxidative phosphorylation demands such as muscle, heart, and brain [21,32].

Acting as the terminal enzyme of the respiratory chain, COX is responsible for the transfer of electrons to molecular oxygen, contributing to the generation of ATP via the proton motive force $[31,33]$. COX15 is one of several accessory proteins not part of the overall complex, but relevant to the structure, synthesis, and processing of COX $[32,34]$. Because COX is a large and intricate complex that is continually being used, assembly proteins are also thought to regulate its activity $[32,35]$. COX is thought to be one of the rate-controlling steps of oxidative phosphorylation [36]. Together, these ideas suggest higher expression of cox 15 could improve the efficiency of respiration. Support for this can be found in the present study in that cells with an insert of cox15 grew faster and produced more recombinant protein (acrp30) than cells without the insert.

Previous studies have shown COX15 is involved in the synthesis of heme a, a compound believed to be critical to energy conservation during oxygen reduction by COX $[33,37,38]$. In fact, defects in the cox 15 gene have resulted in heme a deficiencies whereas defects in COX function have lead to the onset of degenerative diseases such as Leigh syndrome and encephalohepatopathy $[21,32,35]$. To date, only a few cases of these diseases have been the result of a $\operatorname{cox} 15$ mutation; and in those select cases the mutations were heterogeneous suggesting the loss of functional COX15 may be fatal $[21,34]$. It has been proposed that compounds like heme a when bound to proteins, can facilitate the removal of damaging and apoptosis-triggering oxygen radicals $[39,40]$. These observations and proposals suggest that higher cox15 expression might counteract apoptosis, bolstering cell viability. This notion is consistent with findings of the present study where insertion of cox15 resulted in higher viable cell densities than control cells.

The present study has shown the value of comparing fast growing, suspension cells with the slow growing, attached cells using microarrays to uncover genes that may contribute to the enhanced cell growth and improved survival of attached HeLa cells. Two genes were found relevant to enhanced cellular growth and increased viabilities; $c d k l 3$ and cox 15. The proposed functions for these genes, obtained from previous studies, are in agreement with the changes that were observed when over-expressed in several mammalian cell lines. The ability to control the cell cycle and apoptosis can be useful in cell engineering for biotechnology applications by providing greater cell mass and improved yields of recombinant protein $[1,3]$. The comparative physiology studies shown here, provide a useful tool for identifying genes that may serve critical roles in cell growth and other cellular functions. In the present work, a handful of genes were investigated separately, but the potential of this approach (using genomics tools to engineer cell lines) may rely on being able to modulate the expression of many genes simultaneously, rather than individual genes. As such, the present study represents an initial step attempting to directly control cellular features with specific objectives in mind.

\section{Methods}

\section{Bioreactor setup and sampling}

The two HeLa cell lines, attached and suspension were obtained from the American Type Culture Collection (ATCC, Manassas, VA) (Catalog Nos. CCL-2 and CCL-2.2, respectively). Each cell line was grown in a BioFlo 3000 bioreactor (New Brunswick Scientific Co., Edison, NJ) with a working volume of $1.5 \mathrm{~L}$. Both cells lines were grown concurrently. Runs were conducted for up to 7 days after inoculation with constant sampling to characterize growth parameters. Three different runs were carried out for each cell line. The media used was DMEM (Biosource International, Camarillo, CA) supplemented with $10 \%$ FBS (Biosource International, Camarillo, CA). The attached HeLa cells were grown on Cytodex 3 (Amersham Biosciences, Piscataway, NJ) microcarriers. Each reactor was seeded with $2.0 \times 10^{5}$ cells $/ \mathrm{mL}[41,42]$. Cells used to seed the bioreactors were synchronized using serum deprivation for 24 hours $[6,15]$.

Each bioreactor was sampled at regular intervals to test media composition (i.e. pH, Glucose, Lactate), cell viability, and cell density. Each bioreactor was sampled for microarray analysis at the same time corresponding to different phases of growth under batch conditions. Samples were placed in $2 \mathrm{~mL}$ RNase/DNase-free micro tubes (Marsh Biomediacal Products, Rochester, NY), combined with TRIzol reagent (Invitrogen, Carlsbad, CA) and stored at $-80^{\circ} \mathrm{C}$.

\section{RNA isolation and sample preparation}

Total RNA was purified from samples using an Invitrogen kit (Micro-to-Midi Total RNA Purification System) and quantified using a spectrophotometer, GeneQuant Pro (Biochrom Ltd, Cambridge, UK). Absorbance values at $260 \mathrm{~nm}$ and the other at $280 \mathrm{~nm}$ were determined. The quality of RNA was determined by the ratio of these numbers $\left(A_{260 / 280}\right)$. Only samples with an $A_{260 / 280}$ of at least 1.8 were subsequently used for microarray analysis [15]. 
Each sample, consisting of approximately $10 \mu \mathrm{g}$ of total RNA, was reverse transcribed, labeled, and prepared for microarray hybridization using a protocol from The Institute for Genomic Research (TIGR) in Rockville, MD: "Aminoallyl labeling of RNA for Microarrays" (SOP \# M004, Rev. 2) with an effective date of 3/4/2002.

\section{3. cDNA microarray analysis}

Microscope slides (Corning, Corning, NY) were printed with a set of 32,448 spots corresponding to approximately 14,000 unique genes by TIGR (Rockville, MD) using an array fabricator (Intelligent Automation, Rockville, MD). Microarray preparation and hybridization were performed using a protocol "Microarray labeled probe hybridization" (SOP \# M005, Rev. 3) available from TIGR with an effective date of 9/11/2002. After washing and drying, each slide was ready for scanning and image analysis using a GenePix 4000B (Molecular Devices Corporation, Sunnyvale, CA). Both technical and biological replicates were included in the microarray experiments. To ensure proper labeling efficiency the dyes for half of the arrays were swapped $[13,15,43]$.

Acuity software (Molecular Devices Corporation, Sunnyvale, CA) was used to analysis the data starting with total intensity normalization [13]. Additional steps were taken to filter the data, removing spots with either poor signal quality or genes with highly variable (i.e. inconsistent) expression ratios [43]. The expression ratio for a gene, as referred to in the present study, is defined as the test sample (suspension cell line) intensity divided by the control sample (attached cell line) intensity. An expression ratio of unity indicates equal hybridization between the two samples being assayed whereas expression ratios above or below 1.0 are referred to as being upregulated or downregulated, respectively [14].

The clustering algorithms applied to the data included: self-organizing maps (SOMs), principle component analysis (PCA), and hierarchical clustering [14]. Genes clustered together were probed to identify subsets of genes with relevance to cellular growth (i.e. cell cycle regulation, apoptosis, and/or signal transduction). The most differentially expressed were then identified and evaluated based on their known or proposed functionalities as indicated in the literature $[13,15]$.

Microarray results were verified by performing reverse transcription-polymerase chain reaction (RT-PCR) experiments. The protocols used were provided by Applied Biosystems (4333458, Rev. B \& 4335626, Rev. C, both dated 05/2004). Each gene was assayed three times through two separate experiments to establish greater statistical significance.

\section{Enhanced expression of genes in various cell lines}

Sequencing information for both $c d k l 3$ and cox15 were obtained using two public online databases; Harvester (European Molecular Biology Laboratory, Heidelberg, Germany) and GenBank (National Institutes of Health, Bethesda, MD) $[16,17]$. For each gene, a plasmid containing the full-length cDNA was purchased from GeneCopoeia (Germantown, MD). Each plasmid also contained the neomycin gene, conferring resistance to the compound geneticin (Invitrogen, Carlsbad, CA). This resistance allowed for the selection of clones expressing the plasmid in the mammalian cell lines examined $[5,10]$. In subsequent experiments, cells transfected with empty plasmids (i.e. plasmids containing the neomycin gene and other components but neither genes of interest, $c d k l 3$ or cox15) served as the control.

To check gene and protein sequence homologies the Basic Local Alignment Search Tool (BLAST) provided by the National Center for Biotechnology Information (NCBI) (Bethesda, MD) was used. Based on these results, several cell lines were selected for investigation. Each of the following cell lines was purchased from ATCC (Manassas, VA): HEK-293 (Catalog No. CRL-1573), CHO (Catalog No. CCL-61), and MDCK (Catalog No. CCL-34). Another cell line, HEK-293 ACRP30, was also studied because it constitutively expresses acrp30 for which an enzymelinked immunosorbent assay (ELISA) is available $[44,45]$. This cell line was included in subsequent studies to evaluate the impact of $\mathrm{cdkl} 3$ and $\operatorname{cox} 15$ on protein production. Both HEK-293 cell lines were grown in DMEM (Biosource International, Camarillo, CA) with 10\% FBS (Biosource International, Camarillo, $\mathrm{CA}$ ). The media used with the CHO cells was F-12K (ATCC, Manassas, VA) supplemented with 10\% FBS (ATCC, Manassas, VA). The MDCK cells were grown in EMEM (ATCC, Manassas, VA) supplemented with $10 \%$ FBS (ATCC, Manassas, VA). All of these cell lines were grown in an incubator (Thermo Scientific, Waltham, MA) set at $37^{\circ} \mathrm{C}$ and $5 \% \mathrm{CO}_{2}$. In addition, all of the cell lines were dissociated using a trypsin-EDTA solution (ATCC, Manassas, VA).

Once several different cell lines were selected for analysis, subsequent transfections were performed using manufacturer provided protocols packaged with each transfecting agent. For the attached HeLa cells and the HEK-293 cell lines (HEK-293 and HEK-293 ACRP30), the transfecting agent used was Lipofectamine 2000 (Invitrogen, Carlsbad, CA). In contrast, the transfecting agent used for the suspension HeLa cells and the CHO cells was Lipofectamine LTX (Invitrogen, Carlsbad, CA). And for the MDCK cells, Optifect (Invitrogen, Carlsbad, CA) was the transfecting agent used. The transfecting agents were selected based on consultation with the manufacturer, Invitrogen (Carlsbad, CA) in order to maintain consistent 
transfection efficiency levels between cell lines and have reproducible results.

Transfections were performed in 24-well plates (Corning, Corning, NY). Within 24 hours following the transfection the media was replaced with complete media. Once the cells had recovered, usually after 48 hours, geneticin was added to a final concentration of $750 \mu \mathrm{g} / \mathrm{mL}$ based on a prior kill curves (data not shown). Over several days, as media was periodically replaced, colonies began to form. Individual colonies were isolated and moved into 96-well plates (Corning, Corning, NY). Upon reaching confluency, each well was expanded sequentially into 24,12 , and 6-well plates (Corning, Corning, NY) before being counted and screened for expression based on western blot analysis. Physiological changes, post-transfection, were also observed using a DM IRB microscope and attached camera (Leica Camera, Allendale, NJ).

Western blotting was conducted to verify translation of gene inserts via transfected plasmids. Protocols for this method were made available by the manufacturers of the primary antibodies for COX15 (Abnova, Taipei City, Taiwan) and CDKL3 (Abcam, Cambridge, MA). Briefly, cell lysate samples were prepared from a population of cells that were counted and assayed for viability. These samples were diluted with reducing sample buffer and separated using 4-20\% Tris-Glycine gels (Invitrogen, Carlsbad, CA). Proteins were transferred to nitrocellulose membranes (Invitrogen, Carlsbad, CA) and blocked with blotting grade blocker, non-fat dry milk (Bio-Rad, Hercules, CA) for 1 hour. Membranes were then incubated with the primary antibodies at a dilution of 1:500 for 1 hour followed by incubation with secondary antibodies (HRP-conjugated secondary antibodies) for another hour. Incubation with a color development agent (KPL, Gaithersburg, MD) allowed visualization of the blots which were then scanned.

Following clone screening, cells expressing the desired plasmid were expanded successively into 6 -well plates and $25 \mathrm{~cm}^{2}$ T-flasks. For growth comparisons, $75 \mathrm{~cm}^{2}$ T-flasks (Corning, Corning, NY) and $250 \mathrm{~mL}$ spinner flasks (Bellco Glass, Vineland, NJ) were seeded and monitored for $\mathrm{pH}$ (Radiometer Analytical SAS, Lyon, France), cell viability \& density (Cedex HiRes, Innovatis, Malvern, PA), and metabolite concentrations (YSI, Yellow Springs, OH) $[41,42]$. Cell counting was performed two different ways; using a cell counter (Cedex HiRes) and using a hemocytometer. In both cases, duplicates of each sample were assayed. The Cedex HiRes was used as instructed by the manufacturer. Samples counted manually were diluted 1:1 with tryphan blue and counted on a hemocytometer. Variation between duplicates and between counting methods were calculated to be $=2.5 \%$ In conjunction with the spinner flasks, Cytodex 3 microcarriers (GE Healthcare, Piscataway, NJ) were used for the cell lines studied [13]. Cells used for seeding were synchronized using the mitotic shake-off method $[6,9]$.

\section{Flow cytometry and ELISA}

Cells grown in $162 \mathrm{~cm}^{2}$ T-flasks were sampled at different points along their respective growth curves for analysis using a flow cytometer. Samples were taken during the early, middle, and late exponential growth phase, following synchronization by the mitotic shake-off method $[6,9]$. Flow cytometry experiments were performed using a CyAn LX flow cytometer (DakoCytomation, Fort Collins, CO). Cells were fixed with ethanol and stained with propidium iodide (Becton Dickinson, Franklin Lakes, NJ) at a concentration between $0.5-1.0 \times 10^{6}$ cells/mL [46]. Summit software (DakoCytomation, Fort Collins, CO) was used to capture data generated by the sampled cells such as the level of light scattering and fluorescence. In contrast, Modfit software (Molsoft, La Jolla, CA) was used to identify varying stages of the cell cycle and calculate the percentages of cells in each stage.

To quantify the amount of acrp30 secreted by HEK-293 ACRP30 cells an ELISA was performed using a kit produced by R\&D Systems (Catalog No. MRP300, Minneapolis, MN). A microplate spectrophotometer (SpectraMax 190, Molecular Devices, Sunnyvale, CA) was used to quantify the amount of protein present in the samples. Samples were run at varying dilutions to establish the minimum dilution level necessary to obtain values that fall within the standard curve. This value was determined to $1: 4,000$ (data not shown).

\section{Authors' contributions}

PJ was involved in the conception of the work, performed many of the experiments, and drafted portions of the manuscript. $\mathrm{MB}$ conceived part of the work and helped draft the manuscript. KK was involved in the microarray experiments and drafting the manuscript. JS designed several experiments, coordinated much of the work, and drafted portions of the text. All authors read and approved the final manuscript.

\section{Acknowledgements}

Funding was provided by the Intramural program at the National Institute of Diabetes \& Digestive \& Kidney Diseases, National Institutes of Health (NIH) and NIH Grant ROI AR053358.

The authors would like to thank Renee Rubio and Dr. John Quackenbush, formerly at TIGR for their assistance with the cDNA microarrays. The authors would also like to thank Drs. Bruce Raaka and Laertis Ikonomou (Clinical Endocrinology Branch, National Institute of Diabetes \& Digestive \& Kidney Diseases) for their help and guidance with the flow cytometry experiments. The HEK-293 ACRP30 cells were obtained from Dr. Philipp Scherer's laboratory (Department of Cell Biology, Albert Einstein College of Medicine, Yeshiva University). 


\section{References}

I. Seth G, Hossler P, Yee JC, Hu WS: Engineering cells for cell culture bioprocessing-physiological fundamentals. Adv Biochem Eng Biotechnol 2006, I01: I 19-164.

2. Fussenegger M, Bailey JE: Molecular regulation of cell-cycle progression and apoptosis in mammalian cells: implications for biotechnology. Biotechnol Prog 1998, 14:807-833.

3. Bailey JE, Sburlati A, Hatzimanikatis V, Lee K, Renner WA, Tsai PS: Inverse metabolic engineering: a strategy for directed genetic engineering of useful phenotypes. Biotechnol Bioeng 2002, 79:568-579.

4. Barnes LM, Moy N, Dickson AJ: Phenotypic variation during cloning procedures: analysis of the growth behavior of clonal cell lines. Biotechnol Bioeng 2006, 94:530-537.

5. Bohm E, Voglauer R, Steinfellner W, Kunert R, Borth N, Katinger H: Screening for improved cell performance: selection of subclones with altered production kinetics or improved stability by cell sorting. Biotechnol Bioeng 2004, 88:699-706.

6. Merrill GV: Cell synchronization. Methods Cell Biol 1998 57:229-249.

7. Hatzimanikatis $\mathrm{V}$, Lee $\mathrm{KH}$, Bailey JE: A mathematical description of regulation of the GI-S transition of the mammalian cell cycle. Biotechnol Bioeng 1999, 65:631-637.

8. Ifandi $\mathrm{V}, \mathrm{Al}$-Rubeai $\mathrm{M}$ : Stable transfection of $\mathrm{CHO}$ cells with the c-myc gene results in increased proliferation rates, reduces serum dependency, and induces anchorage independence. Cytotechnology 2003, 4I:I-I0.

9. Davis PK, Ho A, Dowdy SF: Biological methods for cell-cycle synchronization of mammalian cells. Biotechniques 2001, 30:1322-1326.

10. Pollard TD, Earnshaw WC: Cell Biology Philadelphia: Saunders; 2004.

II. Masters JR: HeLa cells $\mathbf{5 0}$ years on: the good, the bad and the ugly. Nat Rev Cancer 2002, 2:3 I5-319.

12. Jaluria $P$, Betenbaugh M, Konstantopoulos K, Frank B, Shiloach J: Application of microarrays to identify and characterize genes involved in attachment dependence in HeLa cells. Metab Eng 2007, 9:24I-25I.

13. Burke HB: Discovering Patterns in Microarray Data. Mol Diagn 2000, 5:349-357.

14. Quackenbush J: Computational Analysis of Microarray Data. Nat Rev Genet 2001, 2:418-427.

I5. Simon R, Radmacher MD, Dobbin K: Design of studies using DNA microarrays. Genet Epidemiol 2002, 23:21-26.

16. Harvester [http://harvester.fzk.de/harvester/]

17. GenBank [http://www.ncbi.nlm.nih.gov/]

18. Racher AJ, Moreira JL, Alves PM, Wirth M, Weidle UH, Hauser $\mathrm{H}$ Carrondo MJ, Griffiths JB: Expression of recombinant antibody and secreted alkaline phosphatase in mammalian cell. Influence of cell line and culture system upon production kinetics. Appl Microbiol Biotechnol 1994, 40:85 I-856.

19. Robertson JS: An overview of host cell selection. Dev Biol Stand |999, 98:7-I I.

20. Yee KW, Moore SJ, Midmer M, Zanke BW, Tong F, Hedley D, Minden MD: NKIAMRE, a novel conserved CDC2-related kinase with features of both mitogen-activated protein kinases and cyclin-dependent kinases. Biochem Biophys Res Commun 2003, 308:784-792.

21. Oquendo CE, Antonicka H, Shoubridge EA, Reardon W, Brown GK Functional and genetic studies demonstrate that mutation in the COXI5 gene can cause Leigh syndrome. I Med Genet 2004, $41: 540-544$

22. Midmer M, Haq R, Squire JA, Zanke BW: Identification of NKIAMRE, the human homologue to the mitogen-activated protein kinase-/cyclin-dependent kinase-related protein kinase NKIATRE, and its loss in leukemic blasts with chromosome arm 5q deletion. Cancer Res 1999, 59:4069-4074.

23. Haq R, Randall S, Midmer M, Yee K, Zanke B: NKIATRE is a novel conserved cdc2-related kinase. Genomics 200I, 7|:|3|-|4|.

24. Hanks SK, Quinn AM, Hunter T: The protein kinase family: conserved features and deduced phylogeny of the catalytic domains. Science 1988, 241:42-52.

25. Miyata $Y$, Nishida E: Distantly related cousins of MAP kinase: biochemical properties and possible physiological functions. Biochem Biophys Res Commun 1999, 266:291-295.
26. Hofmann F, Livingston DM: Differential effects of cdk2 and cdk3 on the control of $\mathrm{pBb}$ and E2F function during $\mathbf{G} \mathbf{I}$ exit. Genes Dev 1996, 10:85I-86I.

27. Thompson MA, Stumph J, Henrickson SE, Rosenwald A, Wang $Q$ Olson S, Brandt SJ, Roberts J, Zhang X, Shyr Y, Kinney MC: Differential gene expression in anaplastic lymphoma kinase-positive and anaplastic lymphoma kinase-negative anaplastic large cell lymphomas. Hum Pathol 2005, 36:494-504.

28. Matsuoka M, Matsuura Y, Semba K, Nishimoto I: Molecular cloning of a cyclin-like protein associated with cyclin-dependent kinase 3 (cdk 3) in vivo. Biochem Biophys Res Commun 2000, 273:442-447.

29. Braun K, Holzl G, Soucek T, Geisen C, Moroy T, Hengstschlager M: Investigation of the cell cycle regulation of cdk3-associated kinase activity and the role of cdk3 in proliferation and transformation. Oncogene 1998, I 7:2259-2269.

30. Ren S, Rollins BJ: Cyclin C/cdk3 promotes Rb-dependent G0 exit. Cell 2004, I I 7:239-25I.

31. Petruzzella V, Tiranti V, Fernandez P, lanna P, Carrozzo R, Zeviani M: Identification and characterization of human cDNAs specific to BCSI, PETII2, SCOI, COXI5, and COXII, five genes involved in the formation and function of the mitochondrial respiratory chain. Genomics 1998, 54:494-504.

32. Antonicka H, Mattman A, Carlson CG, Glerum DM, Hoffbuhr KC, Leary SC, Kennaway NG, Shoubridge EA: Mutations in COXI5 produce a defect in the mitochondrial heme biosynthetic pathway, causing early-onset fatal hypertrophic cardiomyopathy. Am J Hum Genet 2003, 72:101-I I4.

33. Glerum DM, Muroff I, Jin C, Tzagoloff A: COXI 5 codes for a mitochondrial protein essential for the assembly of yeast cytochrome oxidase. I Biol Chem 1997, 272:19088-19094

34. Bugiani M, Tiranti V, Farina L, Uziel G, Zeviani M: Novel mutations in COX 15 in a long surviving Leigh syndrome patient with cytochrome c oxidase deficiency. J Med Genet 2005, 42:e28.

35. Pecina P, Houstkova H, Hansikova H, Zeman J, Houstek J: Genetic defects of cytochrome c oxidase assembly. Physiol Res 2004 53(Suppl I):S2I3-S223

36. Groen AK, Wanders RJ, Westerhoff HV, van der Meer R, Tager JM: Quantification of the contribution of various steps to the control of mitochondrial respiration. I Biol Chem 1982 257:2754-2757.

37. Zhuang J, Reddi AR, Wang Z, Khodaverdian B, Hegg EL, Gibney BR: Evaluating the roles of the heme a side chains in cytochrome c oxidase using designed heme proteins. Biochemistry 2006 45:12530-12538.

38. Barros MH, Carlson CG, Glerum DM, Tzagoloff A: Involvement of mitochondrial ferredoxin and Cox $15 p$ in hydroxylation of heme O. FEBS Lett 2001, 492:133-138.

39. Van Houten B, Woshner V, Santos JH: Role of mitochondrial DNA in toxic responses to oxidative stress. DNA Repair (Amst) 2006, 5: $145-152$

40. Kumar S, Bandyopadhyay U: Free heme toxicity and its detoxification systems in human. Toxicol Lett 2005, I57: I75- 88.

4I. Bleckwenn NA, Bentley WE, Shiloach J: Evaluation of production parameters with the vaccinia virus expression system using microcarrier attached HeLa cells. Biotechnol Prog 2005, 2I:554-56I.

42. Bleckwenn NA, Golding H, Bentley WE, Shiloach J: Production of recombinant proteins by vaccinia virus in a microcarrier based mammalian cell perfusion bioreactor. Biotechnol Bioeng 2005, 90:663-674.

43. Hegde P, Qi R, Abernathy K, Gay C, Dharap S, Gaspard R, Hughes JE, Snesrud E, Lee N, Quackenbush J: A concise guide to cDNA microarray analysis. Biotechniques 2000, 29:548-550. 556 passim

44. Mancia F, Patel SD, Rajala MW, Scherer PE, Nemes A, Schieren I, Hendrickson WA, Shapiro L: Optimization of protein production in mammalian cells with a coexpressed fluorescent marker. Structure 2004, I 2: 1355-1360.

45. Berg AH, Combs TP, Du X, Brownlee M, Scherer PE: The adipocyte-secreted protein Acrp30 enhances hepatic insulin action. Nat Med 2001, 7:947-953

46. Darzynkiewicz Z, Juan G: Unit 7.5 DNA content measurement for DNA ploidy and cell cycle analysis. Current Protocols in Cytometry 1997. 